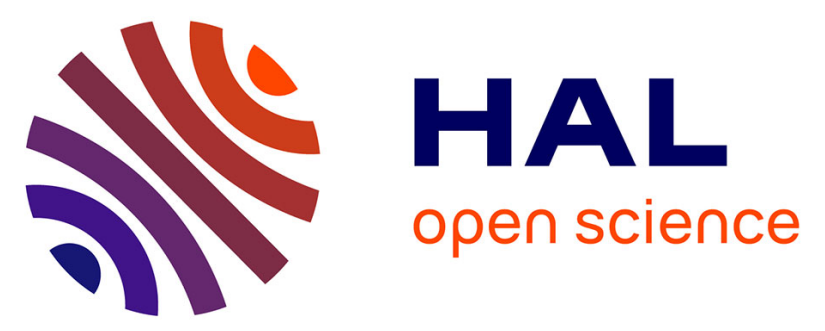

\title{
Open-Circuit Voltage Decay Simulations on Silicon and Gallium Arsenide p-n Homojunctions: Design Influences on Bulk Lifetime Extraction
}

Antoine Lemaire, A. Perona, M. Caussanel, A. Dollet

\section{To cite this version:}

Antoine Lemaire, A. Perona, M. Caussanel, A. Dollet. Open-Circuit Voltage Decay Simulations on Silicon and Gallium Arsenide p-n Homojunctions: Design Influences on Bulk Lifetime Extraction. Microelectronics Journal, 2020, 101, pp.104735. 10.1016/j.mejo.2020.104735 hal-02645548

\author{
HAL Id: hal-02645548 \\ https://hal.science/hal-02645548
}

Submitted on 29 May 2020

HAL is a multi-disciplinary open access archive for the deposit and dissemination of scientific research documents, whether they are published or not. The documents may come from teaching and research institutions in France or abroad, or from public or private research centers.
L'archive ouverte pluridisciplinaire HAL, est destinée au dépôt et à la diffusion de documents scientifiques de niveau recherche, publiés ou non, émanant des établissements d'enseignement et de recherche français ou étrangers, des laboratoires publics ou privés. 


\title{
Open-Circuit Voltage Decay Simulations on Silicon and Gallium Arsenide p-n Homojunctions: Design Influences on Bulk Lifetime Extraction
}

\author{
A. Lemaire ${ }^{a, *}$ A. Perona ${ }^{a}$, M. Caussanel ${ }^{a}$ and A. Dollet ${ }^{b}$ \\ ${ }^{a}$ University of Perpignan Via Domitia, 52 avenue Paul Alduy, 66100, Perpignan, France \\ ${ }^{b}$ PROMES-CNRS laboratory, UPR 8521, Rambla de la thermodynamique, Tecnosud, 66100, Perpignan, France
}

\section{ARTICLE INFO}

\section{Keywords:}

OCVD

Simulations

TCAD Sentaurus

Silicon

Gallium arsenide

Minority charge carriers

Lifetime

Design

\begin{abstract}
A B S T R A C T
Open-Circuit Voltage Decay (OCVD) method was investigated for few decades as a simple and convenient method to characterize effective lifetime into Ge and Si p-n homojunctions. Minority carrier lifetime (MCL) is an important parameter to optimize device design where being able to investigate one type of carriers is an important goal. The p-n homojunction design is of major importance to reach that purpose by OCVD. We carried out Technology Computer Aided-Design simulations of the OCVD signal. The study focused on Si and GaAs p-n homojunctions. We looked into bulk thickness and doping level influences on bulk lifetime extraction. Previously, those influences have not been quantified for Si and never investigated for GaAs. MCL accurate extraction from bulk required a bulk thickness at least 4 times higher than diffusion length and emitter doping levels at least 2 orders of magnitude higher than bulk. Likewise, the paper shows different accuracy of extraction between $\mathrm{GaAs} / \mathrm{Si}$ and $\mathrm{p}$-type/n-type bulk in p-n homojunction.
\end{abstract}

\section{Introduction}

Minority carrier lifetime (MCL) is the most relevant parameter to characterize and optimize electronic and optoelectronic devices such as PV cells [1]. Lifetime depends on material nature and on various treatments the material or device has been subjected to. Downstream, lifetime allows to know material purity and gives a follow-up of process steps quality (etching, passivation, metallization and annealing). Upstream, it is possible to investigate new materials bulk properties. So it enables to optimize design such as bulk thickness and doping levels of a solar cell. Measured lifetime is always the contribution of various and independent electrons-holes recombination mechanisms which are SRH (Shockley-Read-Hall), Auger and radiative type [2]. They occur on either sides of the p-n homojunction and at contact interfaces. Therefore measured lifetime is always an effective lifetime and thus usually represented by the simple Eq. 1 and 2 where $\tau_{\text {eff }}$ is the effective lifetime, $\tau_{\mathrm{SRH}}$ the SRH lifetime, $\tau_{\text {Aug }}$ the Auger lifetime, $\tau_{\text {rad }}$ the radiative lifetime, $\tau_{\mathrm{b}}$ the bulk lifetime and $\tau_{\mathrm{s}}$ the surface lifetime.

$$
\begin{aligned}
\frac{1}{\tau_{\mathrm{eff}}} & =\frac{1}{\tau_{\mathrm{b}}}+\frac{1}{\tau_{\mathrm{s}}} \\
\frac{1}{\tau_{\mathrm{b}}} & =\frac{1}{\tau_{\mathrm{SRH}}}+\frac{1}{\tau_{\mathrm{Aug}}}+\frac{1}{\tau_{\mathrm{rad}}}
\end{aligned}
$$

Since extracted lifetime is always effective, bulk MCL investigation is tough. We decided to investigate the Open-Circuit Voltage Decay (OCVD) method [1] because of its conveniences. First it is a fast and non-destructive method. Then it requires a simple circuit and enables characterization of full devices. Finally, it allows extraction of multiple parameters

$\operatorname{ORCID}(\mathrm{s})$ : such as back surface recombination velocity [3] (combination with short-circuit current decay), level of injection [4, 5], series resistance [6], diffusion length [7] and $V_{\text {capacitance }}$ [8] (corresponds to the voltage when space charge region (SCR) capacitance equals diffusion capacitance). On the other hand, it requires a junction ( $\mathrm{p}-\mathrm{n}$ or Schottky). Therefore an appropriate design (thicknesses and doping levels) has to be defined, in order to highlight the behavior of one type of charge carrier (electron for p-type bulk and hole for n-type bulk) and so to measure only bulk lifetime in our case. We have studied $\mathrm{Si}$ because it has been widely investigated by OCVD. However design effects have never been quantified for this material. On the other hand, OCVD studies on GaAs are absent. Only one experimental study has been published [9] while it is a rather well-known semiconductor. Actually, III/V materials characterized by OCVD are rather scarce $[10,11]$. The aim of this work is to define a p-n homojunction design in order to obtain a better usable OCVD signal. We have worked with TCAD Sentaurus which is a powerful tool to simulate design of devices and circuits. In this paper, influences of emitter doping concentration and bulk thickness of $\mathrm{Si}$ and GaAs p-n homojunctions on bulk MCL extraction by OCVD are evaluated.

\section{OCVD Theory}

OCVD theory was first introduced in 1955, by Gossick [12] and by Lederhandler \& Giacoletto [13] to characterize germanium $(\mathrm{Ge})$. The method is based on the interpretation of Open-Circuit Voltage Decay (OCVD) across a p-n junction after an excitation pulse. The excitation pulse is usually obtained by applying a direct constant bias before opening abruptly the circuit with a switch. Initially, a forward current source was employed as excitation and hereafter optical excitation has been also investigated [14]. Two main 


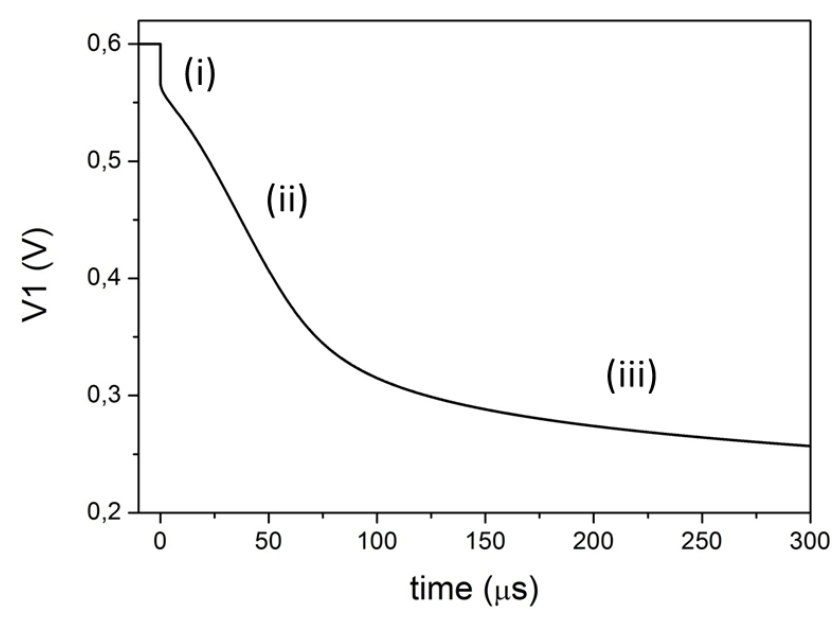

Figure 1: Typical behaviour of OCVD signal in silicon showing usual regions.

circuits can be used: one where switch and characterized diodes are in series $[6,15,16,17]$ (voltage bias source) and the other where they are in parallel $[18,19]$ (current bias source). Usually, OCVD signal presents 3 regions as shown in Fig. 1. The region (i) is due to ohmic resistance [6]. The region (ii) is the one of interest for MCL extraction and is due to minority charge carriers diffusion/recombination. In the ideal case, region (ii) presents a linear decay at low injection level during periods a few times higher than the lifetime. The inverse slope is used into Eq. 3 to calculate effective MCL, where $\eta$ is the $\mathrm{p}$-n junction ideality factor, $k_{\mathrm{B}}$ the Boltzmann constant, $T$ the temperature, $q$ the elementary electric charge and $\mathrm{d} V / \mathrm{d} t$ the linear decay slope. Ideality factor $\eta$ depends on the injection level and on the dominant recombination mechanism [20]. In the region (iii), the voltage variation slows down due to the SCR capacitance [8].

$$
\begin{aligned}
\tau_{\text {eff }} & =-\frac{\eta k_{\mathrm{B}} T}{q} \frac{1}{\mathrm{~d} V / \mathrm{d} t} \\
n(t) & =n_{t 0} \mathrm{e}^{-t / \tau_{\mathrm{eff}}} \\
V(t) & =\frac{k_{\mathrm{B}} T}{q} \ln \left(1+\frac{n(t)}{n_{0}}\right)
\end{aligned}
$$

Eq. 3 is obtained after few assumptions $[12,13]$. First it was assumed lifetime is constant through the bulk. It means p-n junction must be close to an abrupt junction and that recombination centers are homogeneously distributed. Thus density of excess minority carriers decays exponentially with a time constant $\tau_{\text {eff }}$ [21] represented by Eq. 4 where $\mathrm{n}_{t 0}$ represents electrons density at the SCR boundary just before the opencircuit condition. Then, the potential across a p-n junction depends on the logarithm of minority carriers density [20] represented by Eq. 5 where $\mathrm{n}_{0}$ is electron density at thermal equilibrium. Hence it is clear why the theory pretends to obtain a linear region during the decay. Next, the applied voltage has to be much higher than the thermal voltage $\left(V_{\mathrm{a}} \gg \mathrm{V}_{t h}\right)$ to obtain a straight region. Finally, low injection condition must be fulfilled otherwise a second linear region appears

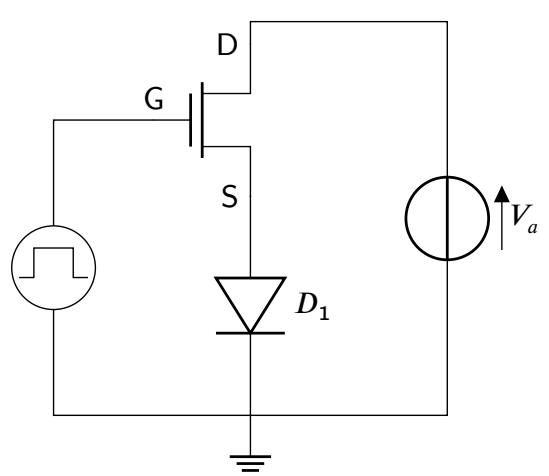

Figure 2: OCVD "series" circuit simulated by TCAD Sentaurus mixed-mode.

and the ideality factor $\eta$ shall be adjusted in consequence (assumed to be around 1 in low injection when SRH recombination is dominant). Experimentally, global OCVD decay can either slow down or speed up according to recombination into the emitter [4] and the SCR [8], to SCR capacitance (also called depletion capacitance) (cf. Fig.1 region (iii)) [8], high injection level [5], low shunt resistance [8] and opencircuiting element [16]. In transient regime, the region (ii) is due to minority charge carriers diffusion/recombination and is represented by the so-called "diffusion capacitance" [8] while region (iii) is due to the "SCR capacitance" driven by majority charge carriers. According to AC equivalent circuit [22] they are in parallel. In consequence linear part is often blurred and so makes MCL extraction tough and unreliable.

\section{Simulation details}

Simulations have been carried out to optimize p-n junction design for bulk MCL extraction by OCVD. The simulated circuit is illustrated by Fig. 2 and represents "series" circuit. This circuit has been selected to perform simulations because of its more convenient configuration and its abundance into literature $[6,15,16,17]$. 2D simulations have been performed with TCAD Sentaurus software (Synopsys) using Sentaurus Device "mixed-mode" [23]. It allows to simulate an entire circuit while modeling one or several components with the finite element method. The characterized p-n junction, so-called D1 (cf. Fig. 2), has been 2D designed (cf. Fig. 3) using Sentaurus Structure Editor (sde) while other components such as MOSFET and voltage sources (cf. Fig. 2) have been selected from well-known SPICE compact models provided by TCAD Sentaurus.

The MOSFET is used to switch on and off the circuit (to reach open-circuit condition across the $p$ - $n$ junction). It is controlled by a pulse generator whereas the $\mathrm{p}-\mathrm{n}$ junction is forward biased by a dc source. MOSFET commutation time has been set up according to our experimental bench ( $24 \mathrm{~ns}$ ). Characterized p-n junctions have a constant and abrupt doping profile. Boron (p-type) / phosphorous (n-type) and beryllium (p-type) / tellurium (n-type) are selected doping species for $\mathrm{Si}$ and GaAs respectively. Bulk doping concentration has been kept equal to $10^{15} \mathrm{~cm}^{-3}$. Naming $x_{\text {emitter }}$ the emitter 


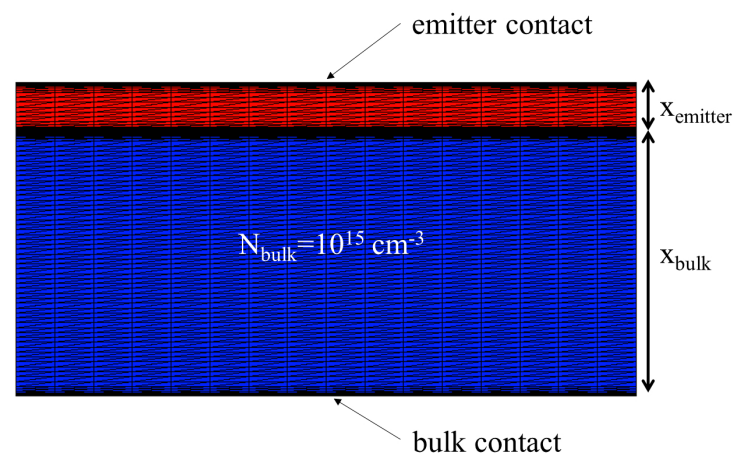

Figure 3: Typical 2D structure of the characterized $p-n$ junction designed in TCAD and used for simulations.

thickness and $L_{\text {emitter }}$ the diffusion length into the emitter, $x_{\text {emitter }} / L_{\text {emitter }}$ ratio has been selected arbitrarily and kept equal to 0.16 . Samples width is $500 \mu \mathrm{m}$. Voltage source has been set up to ensure injection condition into the ohmic regime: it corresponds to $10^{-5} \mathrm{~A}$ for $\mathrm{Si}, 10^{-4} \mathrm{~A}$ for $\mathrm{GaAs}$ p-type bulk and $10^{-2} \mathrm{~A}$ for GaAs n-type bulk.

Poisson and continuity (electrons and holes) equations have been solved using Boltzmann statistics while the DriftDiffusion model has been chosen as the charge carrier transport model. No generation has been considered. SRH, radiative and Auger recombination models have been selected for all simulations. SRH recombination rate is represented by Eq. 6 where $n_{\mathrm{i}}$ is intrinsic charge carrier density, $n_{1}$ and $p_{1}$ statistical factors representing the electron and hole densities for the Fermi level equal to the trap energy level. Indexes " $n$ " and "p" always stand for electrons and holes respectively. We have considered the worst case which is when trap energy level is right in the middle of the band gap. SRH recombination doping dependence follows the Fossum law [24] according to Eq. 7 and 8. Radiative recombination rate is represented by Eq. 9 where $C_{\text {rad }}$, the radiative coefficient, is a constant. The TCAD radiative coefficient for $\mathrm{Si}$ is by default 0 . The Auger recombination rate is represented by Eq. 10 where $C_{\mathrm{n} \text {,Aug }}$ and $C_{\mathrm{p} \text {,Aug }}$ are electron and hole Auger coefficients respectively. The TCAD Auger coefficients for $\mathrm{Si}$ is by default doping dependent while it is not for GaAs. All recombination parameters used for simulations are listed in the Tab. 1 .

$$
\begin{aligned}
R_{\mathrm{SRH}} & =\frac{n p-n_{\mathrm{i}}^{2}}{\tau_{\mathrm{p}_{(\mathrm{TCAD})}}\left(n+n_{1}\right)+\tau_{\mathrm{n}_{(\mathrm{TCAD})}}\left(p+p_{1}\right)} \\
\tau_{\mathrm{n}_{\text {(TCAD })}} & =\frac{\tau_{\mathrm{n}, \max }}{1+\left(N_{\mathrm{A}} / N_{\mathrm{ref}}\right)} \\
\tau_{\mathrm{p}_{(\mathrm{TCAD})}} & =\frac{\tau_{\mathrm{p}, \mathrm{max}}}{1+\left(N_{\mathrm{D}} / N_{\mathrm{ref}}\right)} \\
R_{\mathrm{rad}} & =C_{\mathrm{rad}}\left(n p-n_{\mathrm{i}}{ }^{2}\right) \\
R_{\mathrm{Aug}} & =\left(C_{\mathrm{n}, \mathrm{Aug}} \times n+C_{\mathrm{p}, \mathrm{Aug}} \times p\right)\left(n p-n_{\mathrm{i}}{ }^{2}\right)
\end{aligned}
$$

Electrical contacts are ohmic and insure an infinite surface recombination velocity (SRV) [25]. Actually, TCAD considers electrons and holes with finite maximum velocities $\left(S R V_{\mathrm{n}}=2.573 \times 10^{6} \mathrm{~cm} \mathrm{~s}^{-1}\right.$ and $\left.S R V_{\mathrm{p}}=1.93 \times 10^{6} \mathrm{~cm} \mathrm{~s}^{-1}\right)$ due to thermodynamic considerations. Simulation aim is to extract lifetime through the OCVD signal. Here OCVD response obtained by TCAD simulations has been analyzed according to the following procedure. First step consists in identifying the linear region. Therefore derivative is plotted and so linear region is highlighted through extremum. Then corresponding value is injected into Eq. 3 to get the so-called extracted effective lifetime $\tau_{\text {eff }_{\text {(extracted) }}}$. The local ideality factor $\eta(V)$ [26] is calculated thanks to the dark I-V simulated curve through the following equation:

$$
\eta(V)=\frac{q}{k_{\mathrm{B}} T} \frac{\mathrm{d} V}{\mathrm{~d}(\ln I)}
$$

where $\mathrm{d} V / \mathrm{d}(\ln I)$ is the slope of the $\ln (\mathrm{I})-\mathrm{V}$ curve at the voltage $V$. In our case, the voltage is $V_{\text {ext }}$ where index "ext" stands for extremum. It actually represents the voltage of the extremum identified on the derivative OCVD signal. Finally, extracted value is compared to bulk lifetime value used by TCAD Sentaurus to carry out the simulations and called here TCAD bulk lifetime $\tau_{\text {bulk(TCAD) }}$. Extraction accuracy is directly assessed from this comparison.

\section{Simulation results}

\subsection{Assumptions}

OCVD technique provides effective lifetime. Therefore assumptions must be expressed to investigate the behavior of only one type of charge carrier. Following assumptions have been made to extract lifetime from the bulk. First, bulk thickness must be much longer than diffusion length $\left(L_{\text {bulk }}\right)$ in order to ensure recombination within and to avoid surface recombination at bulk electrical contact. Secondly total current flowing through the diode must be mainly due to the type of carrier under investigation (electrons or holes). Therefore an asymmetric p-n junction is needed $\left(N_{\text {emitter }} \gg N_{\text {bulk }}\right)$. Finally, emitter thickness must be much smaller than the diffusion length $\left(L_{\text {emitter }}\right)$ to prevent recombination within.

\subsection{Bulk thickness influence}

This section studies bulk thickness influence. Bulk is the layer where minority charge carriers under investigation recombine. As previously introduced, its thickness must play an important role to maximize recombination while avoiding surface recombination at bulk electrical contact. Because contacts are considered ohmic, it is of major importance to prevent charge carriers from reaching them. In the reverse case, surface recombination would be disruptive. Indeed, minority carrier density decays exponentially from SCR region through bulk thickness [27]. According to exponential decay law, if the bulk thickness is only once the diffusion length $63 \%$ of minority charge carriers should recombine, $99 \%$ if it is 5 times. Thus OCVD signal has been simulated on abrupt silicon ( $\mathrm{Si}$ ) and gallium arsenide (GaAs) p-n homojunctions with various bulk thicknesses.

Fig. 4 shows [a] OCVD signals, [b] OCVD signals derivatives and $[\mathrm{c}]$ normalized OCVD signal derivatives obtained 
Design influence on lifetime extraction by OCVD

Table 1

Summary of recombination model parameters implemented in TCAD for Si and GaAs.

\begin{tabular}{lcccccc} 
& \multicolumn{2}{c}{ Si } & & \multicolumn{2}{c}{ GaAs } \\
\cline { 2 - 3 } \cline { 5 - 6 } charge carriers & electrons & holes & & electrons & holes \\
\hline$\tau_{\text {max }}[\mathrm{s}]$ & $10^{-5}$ & $10^{16}$ & $3 \times 10^{-6}$ & & $5 \times 10^{-8}$ & $5 \times 10^{-8}$ \\
$N_{\text {ref }}\left[\mathrm{cm}^{-3}\right]$ & & $10^{16}$ & & $10^{16}$ & $10^{16}$ \\
$C_{\text {Aug }}\left[\mathrm{cm}^{6} \mathrm{~s}^{-1}\right]$ & $2.9 \times 10^{-31}-1.3 \times 10^{-30}$ & $1.03-9.52 \times 10^{-31}$ & & $10^{-30}$ & $10^{-30}$ \\
$C_{\text {rad }}\left[\mathrm{cm}^{3} \mathrm{~s}^{-1}\right]$ & 0 & & & $2 \times 10^{-10}$ \\
\hline
\end{tabular}

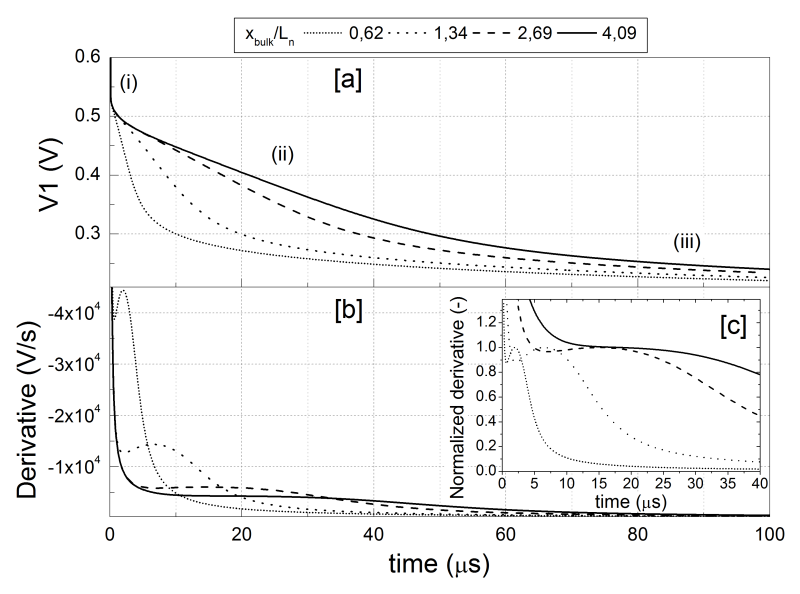

Figure 4: [a] Simulated Open-Circuit Voltage Decay signals, [b] OCVD signal derivatives and [c] Normalized OCVD signal derivatives plotted over time in $\mathrm{Si} p-n$ junctions $\left(N_{\text {emitter }}=N_{\text {bulk }}=10^{15} \mathrm{~cm}^{-3}\right)$ for various p-type bulk thicknesses normalized by electron diffusion length. OCVD signal derivatives have been normalized by their respective extremum value.

by simulation for $\mathrm{Si}$ p-n junctions. GaAs results are given in Fig. 5[a], 5[b] and 5[c]. Homojunctions have symmetrical p-n doping $\left(N_{\text {emitter }}=N_{\text {bulk }}=10^{15} \mathrm{~cm}^{-3}\right)$. The presence of 3 distinguishable regions is highlighted in Fig. 4[a] and 5[a]. The region (i) shows a drastic voltage drop until $520 \mathrm{mV}$ for $\mathrm{Si}$ and $940 \mathrm{mV}$ for $\mathrm{GaAs}$ due to series resistance [6]. This voltage is called $V_{\mathrm{i} \text {-ii }}$ because it represents the voltage transition between the regions (i) and (ii). Due to the difference of their energy band gap, $V_{\mathrm{i} \text {-ii }}$ differs for Si and GaAs. The region (ii), which is the region of interest, presents a rather linear decay until about $300 \mathrm{mV}$ for $\mathrm{Si}$ and $800 \mathrm{mV}$ for GaAs due to minority carriers recombination. This voltage is called $V_{\text {ii-iii }}$ and has been clearly explained by Mahan and Barnes [8]. Once again, this difference between $\mathrm{Si}$ and GaAs is expected because they have such a different lifetime. It means diffusion capacitance of GaAs is much lower than $\mathrm{Si}$ one, while they have rather the same SCR capacitance. The region (iii) proves a drastic slow down due to this SCR capacitance [8]. Fig. 4[b] and 5[b] show 2 extrema in region (ii) that appear at 2 different injection levels: high injection (HI) for a short time and low injection (LI) for a longer time. Fig. 4[c] and $5[\mathrm{c}]$ are plotted to see further extrema. OCVD signal derivatives have been normalized by the second extremum.

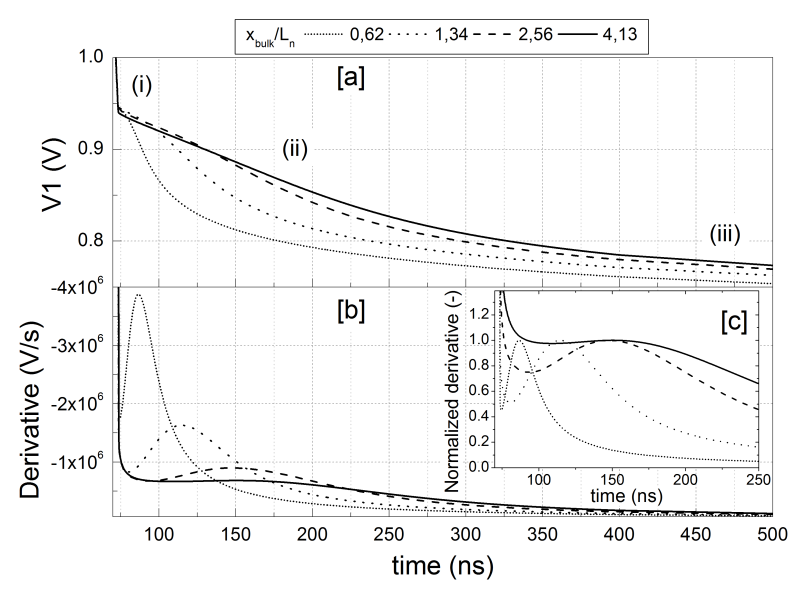

Figure 5: [a] Simulated Open-Circuit Voltage Decay signals, [b] OCVD signal derivatives and [c] Normalized OCVD signal derivatives plotted over time in $\mathrm{GaAs}$ p-n junctions $\left(N_{\text {emitter }}=N_{\text {bulk }}=10^{15} \mathrm{~cm}^{-3}\right)$ for various p-type bulk thicknesses normalized by electron diffusion length. OCVD signal derivatives have been normalized by their respective extremum value.

Since total recombination rate is roughly SRH recombination rate for $\mathrm{HI}$ and $\mathrm{LI}, \mathrm{SRH}$ recombination can be considered as the dominant mechanism influencing minority charge carrier behaviour into the bulk. SRH recombination theory exposed in 1952 by Shockley Read [28] and Hall [29] demonstrates that in low injection level bulk lifetime depends only on one charge carrier type (cf. Eq. 12) while in high injection level it depends on both charge carrier type (cf. Eq. 13).

$$
\begin{aligned}
\tau_{\text {bulk }_{\mathrm{SRH}}(\mathrm{LI})} & =\tau_{\mathrm{n}} \\
\tau_{\text {bulk }_{\mathrm{SRH}}(\mathrm{HI})} & =\tau_{\mathrm{n}}+\tau_{\mathrm{p}}
\end{aligned}
$$

These two distinguishable regions are more visible for GaAs and tend to fuse when bulk thickness increases. The higher the thickness the longer the linear decay or plateau duration. It signifies the extraction becomes more accurate. We have defined plateau duration $\Delta t_{\text {plateau }}$ as time when the $\mathrm{d} V / \mathrm{d} t$ extremum varies about $\pm 5 \%$. Indeed, for $\mathrm{Si}$ it is $19.2 \mu \mathrm{s}(\approx$ $\left.2.1 \tau_{\text {bulk(TCAD })}\right)$ while it is about $71.9 \mathrm{~ns}\left(\approx 1.6 \tau_{\text {bulk(TCAD })}\right)$ for GaAs. We observe that extrema position ( $\left.t_{\text {extremum }}\right)$ shifts toward longer times when thickness increases. This delayed phenomenon can be related to the increase of circuit RC constant because of bulk resistance increasing. The main differences between Si Fig. 4 and GaAs Fig. 5 are voltages 


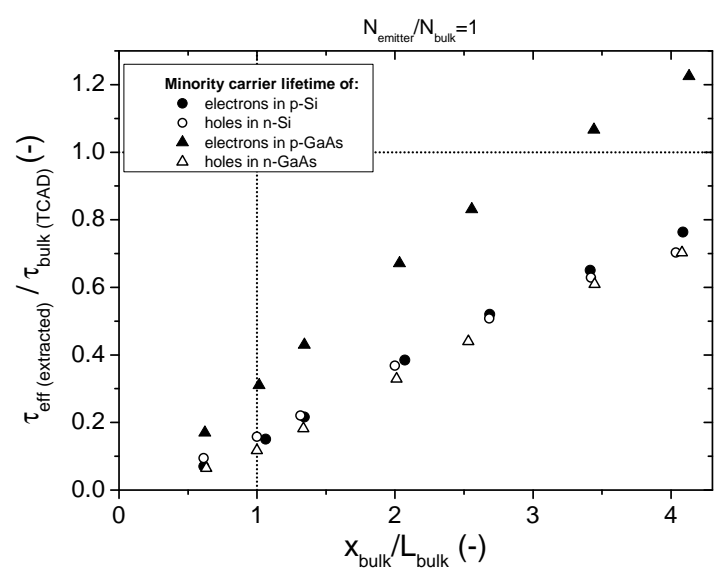

Figure 6: Normalized extracted lifetime plotted over normalized bulk thickness in Si (circle) and GaAs (triangle) p-n homojunctions from OCVD simulations. Full symbols represent electrons in p-type bulk and empty symbols holes in n-type bulk. $\tau_{\text {bulk(TCAD) }}$ is the bulk lifetime used by TCAD and $L_{\text {bulk }}$ is the bulk diffusion length.

of transition regime $\left(V_{\mathrm{i}-\mathrm{ii}}\right.$ and $\left.V_{\mathrm{ii} \text {-iii }}\right)$ and scale of the total decay time. It is shorter for GaAs than for Si. Indeed, bulk lifetime is much lower for GaAs.

Fig. 6 shows extracted effective lifetime to TCAD bulk lifetime ratio plotted over bulk thickness to bulk diffusion length ratio for $\mathrm{Si}$ and $\mathrm{GaAs}$ p-n homojunctions where bulk diffusion length is constant for a given doping level. These two normalized axis allow to compare any materials. It shows results for $\mathrm{Si}$ (circle) and GaAs (triangle) p-n junctions in p-type (full symbols) and n-type bulk (empty symbols). Extracted effective lifetimes have been calculated for LI so we have considered the second extremum from derivative $\mathrm{d} V / \mathrm{d} t$ Fig. 4[b], [c] and 5[b], [c]. In both Si and GaAs case, extracted effective lifetimes increase with the bulk thickness and reach a maximum value for $x_{\text {bulk }}>4.1 L_{\text {bulk }}$. When thickness is smaller than diffusion length it clearly shows surface recombination influence at bulk electrical contact. The higher the minority charge carrier density at the contact the lower the surface lifetime. Moreover this maximum value gets closer to the TCAD bulk lifetime with the exception of electrons lifetime in GaAs. Indeed, MCL is overestimated around $22.5 \%$ while it is underestimated around $30 \%$ for holes lifetime in both materials and $24 \%$ for electrons lifetime in $\mathrm{Si}$. This trend could be attributed to the higher mobility imbalance between holes and electrons in GaAs.

\subsection{Doping asymmetry influence}

In this section is investigated doping asymmetry influence in p-n homojunction. As previously introduced, a heavily doped emitter is required to extract bulk lifetime accurately. A $\mathrm{p}-\mathrm{n}^{+}$or $\mathrm{n}-\mathrm{p}^{+}$homojunction configuration is essential to extract accurately by OCVD electrons or holes lifetime respectively. This configuration should lower the weight of minority charge carriers which are not under investigation.

Fig. 7 and 8 show [a] OCVD signals obtained by simulation and [b] OCVD signal derivatives for $\mathrm{Si}$ and $\mathrm{GaAs}$

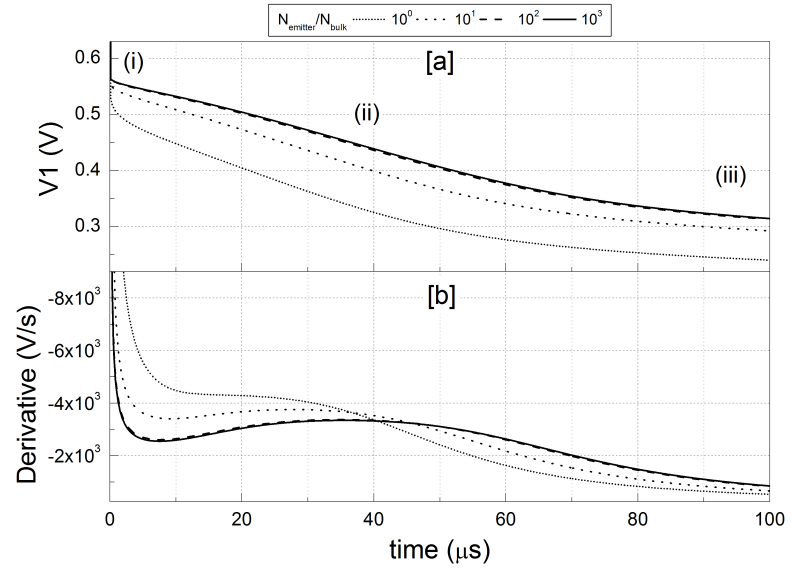

Figure 7: [a] Simulated Open-Circuit Voltage Decay signals, [b] OCVD signal derivatives plotted over time in Si p-n homojunctions for emitter (n-type) doping level equal to $10^{15} \mathrm{~cm}^{-3}$ up to $10^{18} \mathrm{~cm}^{-3}$ and bulk (p-type) doping level equal to $10^{15} \mathrm{~cm}^{-3}$. $x_{\text {bulk }} \approx 4.1 L_{\text {bulk }}$.

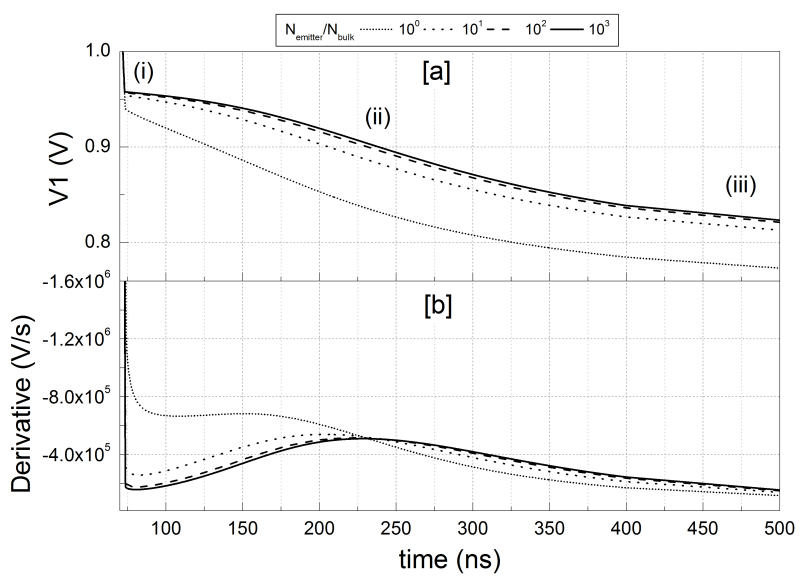

Figure 8: [a] Simulated Open-Circuit Voltage Decay signals, [b] OCVD signal derivatives plotted over time in GaAs p-n homojunctions for emitter (n-type) doping level equal to $10^{15} \mathrm{~cm}^{-3}$ up to $10^{18} \mathrm{~cm}^{-3}$ and bulk (p-type) doping level equal to $10^{15} \mathrm{~cm}^{-3}$. $x_{\text {bulk }} \approx 4.1 L_{\text {bulk }}$.

p-n homojunctions respectively. Bulk doping level is kept constant and equal to $10^{15} \mathrm{~cm}^{-3}$ while emitter doping level varies from $10^{15} \mathrm{~cm}^{-3}$ up to $10^{18} \mathrm{~cm}^{-3}$. Bulk thickness over bulk diffusion length is about 4.1. We still observe 3 different regions in Fig. 7[a] and 8[a]: regions (i) still show a drastic voltage drop where $V_{\mathrm{i} \text {-ii }}$ is roughly $560 \mathrm{mV}$ and $960 \mathrm{mV}$ for $\mathrm{Si}$ and $\mathrm{GaAs}$ respectively. It increases slightly with emitter doping level because of built-in voltage rising; regions (ii) also present a rather linear decay due to minority charge carriers diffusion/recombination where $V_{\text {ii-iii }}$ goes up from $300 \mathrm{mV}$ to $350 \mathrm{mV}$ for $\mathrm{Si}$ and from $800 \mathrm{mV}$ to $850 \mathrm{mV}$ for GaAs. This is due to the asymmetric junction which reduces the absolute SCR thickness and thus faintly increases the SCR capacitance. Therefore it reduces the region (ii) duration and hence the extraction accuracy but it is actually compensated by the rise of $V_{\text {i-ii }}$. Two extrema are appearing 


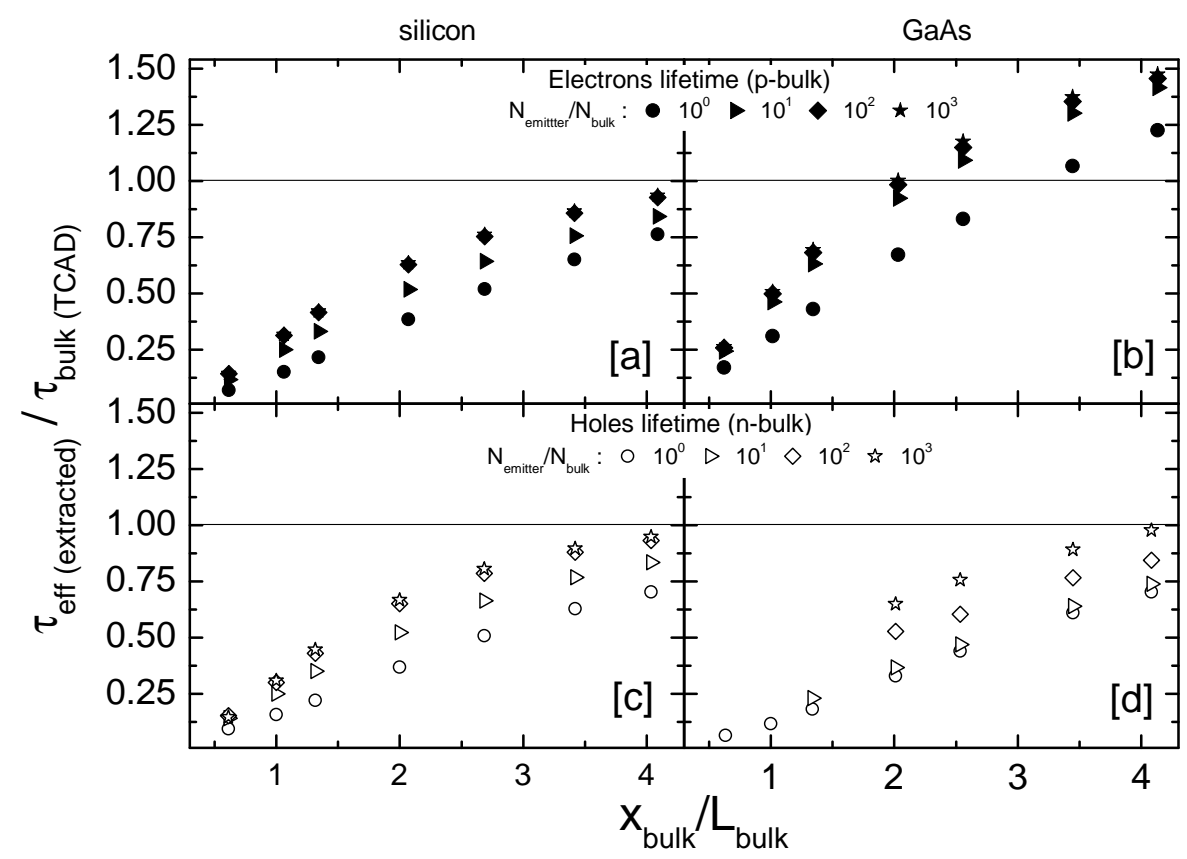

Figure 9: [a] [b] normalized extracted lifetime plotted over normalized bulk thickness in Si p-n homojunction and [c] [d] in GaAs p-n homojunction from OCVD simulations. [a] [c] represent electrons (full symbols) in p-type bulk and [b] [d] holes (empty symbols) in n-type bulk. $\tau_{\text {bulk(TCAD) }}$ is the bulk lifetime used by TCAD and $L_{\text {bulk }}$ is the bulk diffusion length. Each symbol type stand for specific emitter doping level.

on Fig. 7[b] and $8[\mathrm{~b}]$ by increasing emitter doping level. The first is due to HI and the second to LI. This effect is more evident for GaAs. Therefore it reduces LI plateau length in the case of GaAs. Indeed, $\Delta t_{\text {plateau }}$ varies with emitter doping from $71.9 \mathrm{~ns}$ to $63.3 \mathrm{~ns}$ for $\mathrm{GaAs}$ and from $19.2 \mu$ s to $24.1 \mu$ s for $\mathrm{Si}$. This difference could be attributed to higher injection level in the case of GaAs homojunction. Thus injection level is an important parameter because the longer the plateau duration the better the lifetime extraction. Derivative plateau is shifted to a longer time for both Si and GaAs. However there is no significant emitter doping level influence for $N_{\text {emitter }} / N_{\text {bulk }}>10^{2}$ in Fig. 7 and 8.

Fig. 9 shows the extracted effective lifetime to TCAD bulk lifetime ratio plotted over bulk thickness to bulk diffusion length ratio where main results are summarized in Tab.2. Local ideality factors extracted to calculate $\tau_{\text {eff (extracted) }}$ through Eq. 3 are given in Tab.2. They are rather close to unity for $\mathrm{Si}$ while they are higher for GaAs. It expresses the level injection moving which is more prominent in the case of GaAs. Fig. 9[a],[c] shows results for Si and [b],[d] for GaAs p-n homojunctions. Moreover full symbols represent p-type bulk (Fig. 9[a] and [b]) while empty symbols n-type bulk (Fig. $9[\mathrm{c}]$ and [d]). Emitter doping level has been increased by several orders of magnitude from $10^{15} \mathrm{~cm}^{-3}$ to $10^{18} \mathrm{~cm}^{-3}$ while bulk doping level has been kept constant and equal to $10^{15} \mathrm{~cm}^{-3}$. Fig. 9 analysis points out that all extracted effective lifetimes for both materials increase and tend to reach a constant value with increasing bulk thickness. Furthermore we can clearly see the influence of doping asymmetrization. Lifetime extraction becomes closer to TCAD bulk lifetime
Table 2

Summary of extracted results from TCAD simulations with $x_{\text {bulk }} / L_{\text {bulk }} \approx 4.1$ and $N_{\text {emitter }} / N_{\text {bulk }}=10^{3}$

\begin{tabular}{|c|c|c|c|}
\hline & bulk type & $\mathrm{Si}$ & $\mathrm{GaAs}$ \\
\hline$x_{\text {bulk }}[\mu \mathrm{m}]$ & $\begin{array}{l}\text { n-doped } \\
\text { p-doped }\end{array}$ & $\begin{array}{l}730 \\
230\end{array}$ & $\begin{array}{c}126 \\
29\end{array}$ \\
\hline$L_{\text {bulk }}[\mu \mathrm{m}]$ & $\begin{array}{l}/ / \\
/ /\end{array}$ & $\begin{array}{c}179 \\
57\end{array}$ & $\begin{array}{c}31 \\
7\end{array}$ \\
\hline$\tau_{\text {bulk(TCAD) }}[\mathrm{s}]$ & $\begin{array}{l}1 / \\
/ /\end{array}$ & $\begin{array}{l}9.09 \times 10^{-6} \\
2.73 \times 10^{-6}\end{array}$ & $\begin{array}{l}4.55 \times 10^{-8} \\
4.55 \times 10^{-8}\end{array}$ \\
\hline$\tau_{\text {eff(extracted) }}[\mathrm{s}]$ & $\begin{array}{l}/ / \\
/ /\end{array}$ & $\begin{array}{l}8.49 \times 10^{-6} \\
2.58 \times 10^{-6}\end{array}$ & $\begin{array}{l}6.70 \times 10^{-8} \\
4.44 \times 10^{-8}\end{array}$ \\
\hline error [\%] & $\begin{array}{l}/ / \\
/ /\end{array}$ & $\begin{array}{l}-6.5 \\
-5.3\end{array}$ & $\begin{array}{l}+47.3 \\
-2.3\end{array}$ \\
\hline$t_{\text {extremum }}\left[\tau_{\text {bulk(TCAD) }}\right]$ & $\begin{array}{l}1 / \\
/ /\end{array}$ & $\begin{array}{l}3.89 \\
5.78\end{array}$ & $\begin{array}{l}3.40 \\
3.98\end{array}$ \\
\hline$\Delta t_{\text {plateau }}\left[\tau_{\text {bulk(TCAD) }}\right]$ & $\begin{array}{l}1 / \\
/ /\end{array}$ & $\begin{array}{l}2.65 \\
2.38\end{array}$ & $\begin{array}{l}1.39 \\
1.68\end{array}$ \\
\hline$\eta\left(V_{e x t}\right)[-]$ & $1 /$ & $\begin{array}{l}1.10 \\
1.05\end{array}$ & $\begin{array}{l}1.32 \\
1.21\end{array}$ \\
\hline
\end{tabular}

and so more accurate when doping asymmetry increases. Actually, there is no significant variation for more than 2 orders of magnitude between emitter and bulk doping levels in Si. This put forward the minimum doping ratio required to get 
wished asymmetry effect. However, there are tiny nuances for lifetime extraction in GaAs. On the one hand, there is still a significant variation between $N_{\text {emitter }} / N_{\text {bulk }}=10^{2}$ and $10^{3}$ for holes lifetime extraction Fig. 9[d]. Moreover, extractions for $x_{\text {bulk }}<1.3 L_{\text {bulk }}$ have not been carried out because linear regions were not identifiable. On the other hand, there is no massive effect of the asymmetry when $N_{\text {emitter }} / N_{\text {bulk }}>10^{1}$ for electrons lifetime extraction Fig. 9[b]. This discrepancy is due to the higher imbalance mobility between holes and electrons in GaAs $\left(\mu_{\mathrm{n}} / \mu_{\mathrm{p}}=18\right)$ than in $\mathrm{Si}\left(\mu_{\mathrm{n}} / \mu_{\mathrm{p}}=3\right)$. Indeed, this induces that GaAs p-n junction is intrinsically asymmetric when $N_{\text {emitter }}=N_{\text {bulk }}$. Therefore, the higher the imbalance mobility, the higher the required asymmetric ratio.

\section{Conclusion}

OCVD simulations have been performed with TCAD Sentaurus to investigate design influence on bulk lifetime extraction. Extraction has been carried out by identifying linear voltage decay through $\mathrm{d} V / \mathrm{d} t$ evolution. Bulk thickness and doping levels asymmetry between the bulk and the emitter have been considered as the major influence design parameters through study of $\mathrm{Si}$ and GaAs p-n homojunctions. Electrons (in p-type bulk) and holes (in n-type bulk) lifetime extractions have been carried out successfully in $\mathrm{Si}$ and GaAs. OCVD simulations results enable to extract the bulk lifetime with the accuracy of $-6.5 \%$ and $-5.3 \%$ in Si homojunction while $+47.3 \%$ and $-2.3 \%$ in GaAs homojunction for electrons and holes respectively. This results have been obtained with the help of simulated dark I-V curves in order to get the local ideality factor. These results have been reached with a ratio $x_{\text {bulk }} / L_{\text {bulk }} \approx 4.1, N_{\text {emitter }} / N_{\text {bulk }}=10^{3}$ and $x_{\text {emitter }} / L_{\text {emitter }} \approx 0.16$. Initially it has been illustrated by simulation that the thicker the bulk the better the extraction. First because $\Delta t_{\text {plateau }}$ increases and secondly because extracted lifetime gets closer to TCAD bulk lifetime and so becomes more accurate. Indeed, this configuration avoids surface recombination at bulk contact. Since electrical contacts have been considered ohmic, their OCVD signal contribution is not negligible. Indeed, ohmic contact represents infinite surface recombination velocity. Moreover it has been illustrated that plateau is shifted to a longer time, surely due to circuit RC constant rise. Finally, it has been demonstrated by simulation that emitter doping level should be at least 2 orders of magnitude higher than bulk doping level to get the best extraction accuracy. However, this ratio of 2 may be lower/higher depending on the mobility imbalance $\left(\mu_{\mathrm{n}} / \mu_{\mathrm{p}}\right)$ of the semiconductor studied. Difference of MCL extraction accuracy between electrons and holes is lower in $\mathrm{Si}$ than in GaAs. Electrons lifetime extraction is overestimated while holes one is underestimated in GaAs. This could be attributed to the higher mobility and SRV discordance between holes and electrons in GaAs. Our study has well confirmed and quantified our assumptions. It has also initiated research on GaAs OCVD behaviour.

\section{Acknowledgment}

This work was supported financially by PPCM and COSMIC teams from PROMES-CNRS laboratory.

\section{References}

[1] D. K. Schroder, Semiconductor material and device characterization, 3 ed., Wiley-Interscience, Hoboken, NJ, 2006. OCLC: 254501000.

[2] D. K. Schroder, Carrier lifetimes in silicon, IEEE transactions on Electron Devices 44 (1997) 160-170.

[3] B. H. Rose, Minority-carrier lifetime measurements on silicon solar cells using I sc and V oc transient decay, IEEE Transactions on Electron Devices 31 (1984) 559-565.

[4] M. A. Green, Solar cell minority carrier lifetime using open-circuit voltage decay, Solar cells 11 (1984) 147-161.

[5] S. C. Jain, U. C. Ray, R. Muralidharan, V. K. Tewary, Open circuit voltage decay in $\mathrm{p}-\mathrm{n}$ junction diodes at high levels of injection, SolidState Electronics 29 (1986) 561-570.

[6] M. A. Green, Minority carrier lifetimes using compensated differental open circuit voltage decay, Solid-state electronics 26 (1983) 11171122 .

[7] U. Stutenbaeumer, E. Lewetegn, Comparison of minority carrier diffusion length measurements in silicon solar cells by the photoinduced open-circuit voltage decay (OCVD) with different excitation sources, Renewable energy 20 (2000) 65-74.

[8] J. E. Mahan, D. L. Barnes, Depletion layer effects in the open-circuitvoltage-decay lifetime measurement, Solid-State Electronics 24 (1981) 989-994.

[9] H. G. Bhimnathwala, S. D. Tyagi, S. Bothra, S. K. Ghandi, J. M. Borrego, Lifetime measurements by open circuit voltage decay in GaAs and InP diodes, in: Photovoltaic Specialists Conference, 1990., Conference Record of the Twenty First IEEE, IEEE, 1990, pp. 394 398.

[10] A. Zemel, M. Gallant, Carrier lifetime in InP/InGaAs/InP by opencircuit voltage and photoluminescence decay, Journal of Applied Physics 78 (1995) 1094-1100.

[11] P. Dalapati, N. B. Manik, A. N. Basu, Effect of temperature on the intensity and carrier lifetime of an $\mathrm{AlGaAs}$ based red light emitting diode, Journal of Semiconductors 34 (2013) 092001.

[12] B. R. Gossick, On the Transient Behavior of Semiconductor Rectifiers, Journal of Applied Physics 26 (1955) 1356-1365.

[13] S. R. Lederhandler, L. J. Giacoletto, Measurement of minority carrier lifetime and surface effects in junction devices, Proceedings of the IRE 43 (1955) 477-483.

[14] J. E. Mahan, T. W. Ekstedt, R. I. Frank, R. Kaplow, Measurement of minority carrier lifetime in solar cells from photo-induced opencircuit voltage decay, IEEE Transactions on Electron devices 26 (1979) $733-739$.

[15] M. Caussanel, A. Canals, S. K. Dixit, M. J. Beck, A. D. Touboul, R. D. Schrimpf, D. M. Fleetwood, S. T. Pantelides, Doping-Type Dependence of Damage in Silicon Diodes Exposed to X-Ray, Proton, and $\mathrm{He}^{\wedge}\{+\} \$$ Irradiations, IEEE Transactions on Nuclear Science 54 (2007) 1925-1930.

[16] S. Bellone, G. Licciardo, An Analog Circuit for Accurate OCVD Measurements, IEEE Transactions on Instrumentation and Measurement 57 (2008) 1112-1117. 
[17] O. Marcelot, P. Magnan, Comparison between TCAD simulated and measured carrier lifetimes in CMOS photodiodes using the Open Circuit Voltage Decay method, Solid-State Electronics 81 (2013) 135139.

[18] M. P. Deshmukh, R. A. Kumar, J. Nagaraju, Measurement of solar cell ac parameters using the time domain technique, Review of scientific instruments 75 (2004) 2732-2735.

[19] P. C. Ariel, M. A. Cappelletti, E. L. P. y Blanca, A computerized method for carrier lifetime measurement in PN junctions at high and low-level injection, in: 2010 Argentine School of MicroNanoelectronics, Technology and Applications (EAMTA), IEEE, 2010, pp. 87-93.

[20] S. M. Sze, K. K. Ng, Physics of semiconductor devices, 3 ed., WileyInterscience, Hoboken, NJ, 2007. OCLC: 255534639.

[21] Y. R. Nosov, Switching in Semiconductor Diodes, Monographs in Semiconductor Physics, Springer US, 1969.

[22] R. Salach-Bielecki, T. Pisarkiewicz, T. Stapinski, P. Wójcik, Influence of junction parameters on the open circuit voltage decay in solar cells, Opto-Electron. Rev 12 (2004) 79-83.

[23] Synopsys, Device Simulation, 2019. URL: https://www. synopsys.com/ silicon/tcad/device-simulation. html.

[24] J. G. Fossum, Computer-aided numerical analysis of silicon solar cells, Solid-State Electronics 19 (1976) 269-277.

[25] A. G. Aberle, Surface passivation of crystalline silicon solar cells: a review, Progress in Photovoltaics: Research and Applications 8 (2000) 473-487.

[26] K. R. McIntosh, Lumps, Humps and Bumps: Three Detrimental E ects in the Current \{Voltage Curve of Silicon Solar Cells (2001) 190.

[27] H. Mathieu, H. Fanet, Physique des semiconducteurs et des composants électroniques-6ème édition: Cours et exercices corrigés, Dunod, 2009.

[28] W. Shockley, W. T. Read Jr, Statistics of the recombinations of holes and electrons, Physical review 87 (1952) 835.

[29] R. N. Hall, Electron-hole recombination in germanium, Physical review 87 (1952) 387. 